\title{
Maximum superheating and undercooling: Systematics, molecular dynamics simulations, and dynamic experiments
}

\author{
Sheng-Nian Luo* and Thomas J. Ahrens \\ Lindhurst Laboratory of Experimental Geophysics, Seismological Laboratory, California Institute of Technology, Pasadena, \\ California 91125, USA
}

Tahir Çağın, Alejandro Strachan, and William A. Goddard III

Materials and Process Simulation Center, Beckman Institute, California Institute of Technology, Pasadena, California 91125, USA

Damian C. Swift

P-24 Plasma Physics, Los Alamos National Laboratory, Los Alamos, New Mexico 87545, USA

(Received 18 June 2003; published 14 October 2003)

\begin{abstract}
The maximum superheating and undercooling achievable at various heating (or cooling) rates were investigated based on classical nucleation theory and undercooling experiments, molecular dynamics (MD) simulations, and dynamic experiments. The highest (or lowest) temperature $T_{c}$ achievable in a superheated solid (or an undercooled liquid) depends on a dimensionless nucleation barrier parameter $\beta$ and the heating (or cooling) rate $Q . \beta$ depends on the material: $\beta \equiv 16 \pi \gamma_{s l}^{3} /\left(3 k T_{m} \Delta H_{m}^{2}\right)$ where $\gamma_{s l}$ is the solid-liquid interfacial energy, $\Delta H_{m}$ the heat of fusion, $T_{m}$ the melting temperature, and $k$ Boltzmann's constant. The systematics of maximum superheating and undercooling were established phenomenologically as $\beta=\left(A_{0}-b \log _{10} Q\right) \theta_{c}\left(1-\theta_{c}\right)^{2}$ where $\theta_{c}=T_{c} / T_{m}, A_{0}=59.4, b=2.33$, and $Q$ is normalized by $1 \mathrm{~K} / \mathrm{s}$. For a number of elements and compounds, $\beta$ varies in the range $0.2-8.2$, corresponding to maximum superheating $\theta_{c}$ of $1.06-1.35$ and $1.08-1.43$ at $Q$ $\sim 1$ and $10^{12} \mathrm{~K} / \mathrm{s}$, respectively. Such systematics predict that a liquid with certain $\beta$ cannot crystallize at cooling rates higher than a critical value and that the smallest $\theta_{c}$ achievable is $1 / 3$. MD simulations $(Q$ $\left.\sim 10^{12} \mathrm{~K} / \mathrm{s}\right)$ at ambient and high pressures were conducted on close-packed bulk metals with Sutton-Chen many-body potentials. The maximum superheating and undercooling resolved from single- and two-phase simulations are consistent with the $\theta_{c}-\beta-Q$ systematics for the maximum superheating and undercooling. The systematics are also in accord with previous MD melting simulations on other materials (e.g., silica, Ta and $\epsilon$-Fe) described by different force fields such as Morse-stretch charge equilibrium and embedded-atom-method potentials. Thus, the $\theta_{c}-\beta-Q$ systematics are supported by simulations at the level of interatomic interactions. The heating rate is crucial to achieving significant superheating experimentally. We demonstrate that the amount of superheating achieved in dynamic experiments $\left(Q \sim 10^{12} \mathrm{~K} / \mathrm{s}\right)$, such as planar shock-wave loading and intense laser irradiation, agrees with the superheating systematics.
\end{abstract}

DOI: $10.1103 / P h y s R e v B .68 .134206$

PACS number(s): 64.60.Qb, 64.70.Dv, 62.50.+p

\section{INTRODUCTION}

The melting of crystals and crystallization of liquids are of scientific and technological significance. Metastable superheating and undercooling are inherent in melting and freezing processes. A fundamental issue of immediate theoretical and experimental interest is the extent to which a solid can be superheated and a liquid undercooled. Numerous undercooling experiments have been conducted to probe the maximum undercooling on elements and compounds, ${ }^{1}$ and appreciable undercooling has been observed as homogeneous nucleation of crystals from the liquid is readily attainable experimentally. However, considerable superheating has rarely been achieved except for a few cases with special experimental designs, ${ }^{2}$ due to the fact that heterogeneous nucleation is dominant in most melting experiments with low heating rates. Heterogeneous nucleation of the melt is favored at sites with free surfaces, defects, and impurities which significantly lower the energy barriers for nucleation. Predicting the maximum superheating is particularly challenging due to a paucity in experimental data.

Shock-state sound-speed and temperature measurements on metals, alkali halides, and silicates demonstrate nonequilibrium melting. Superheating has been proposed to explain the sharp drop of temperature at successive shock states as pressure is increased along the Hugoniot. ${ }^{3-5}$ Similarly, nonequilibrium melting has been observed in intense laser irradiation. ${ }^{6}$ Considerable superheating achieved in ultrafast dynamic loading (heating rate $Q \sim 10^{9}-10^{12} \mathrm{~K} / \mathrm{s}$ ) and its rarity in conventional melting experiments $(Q \sim 1 \mathrm{~K} / \mathrm{s})$ indicate the important role of heating rates in achieving superheating. The melting temperatures obtained previously from shockwave experiments ${ }^{5,7}$ are significantly higher than those extrapolated from recent diamond-anvil-cell (DAC) data ${ }^{8}$ for transition metals such as Fe, V, Mo, W, and Ta. These discrepancies raise such issues as the interpretation of temperature measurements in shock melting experiments and the maximum superheating achievable at various heating rates.

Molecular dynamics (MD) simulation is a useful tool to study melting and freezing processes under various pressures. It has long been recognized that temperature hysteresis exists in MD simulations of bulk crystal with threedimensional (3D) periodic boundaries. ${ }^{9-13}$ But a systematic and quantitative investigation of both superheating and un- 
dercooling in MD simulations has not been previously conducted. It is of particular interest whether consistent predictions of both superheating and undercooling and also material properties can be made using a single set of force fields in MD simulations. Heating (cooling) rates typical in conventional MD simulations are on the order of $10^{12} \mathrm{~K} / \mathrm{s}$, comparable to light-gas-gun shock-wave experiments and intense laser irradiation. Thus melting and freezing simulations with MD can be checked against ultrafast dynamic experiments and the predictions of various superheating theories.

Previously, theoretical models for melting are mostly based on the Lindemann's vibration criterion ${ }^{14}$ and Born's shear instability. ${ }^{15,16}$ The limit of superheating has been studied assuming the catastrophes of entropy, ${ }^{17}$ rigidity, and volume $^{18}$ upon melting. Other efforts to describe superheating utilized kinetic nucleation theory. ${ }^{19,20}$ Recently, we proposed a systematic framework to predict the maximum superheating (and undercooling) at various heating (cooling) rates. ${ }^{21}$ In this work, we extend our previous efforts ${ }^{21}$ to present detailed treatments of the systematics and MD simulations of the maximum undercooling and superheating and dynamic melting experiments. Section II establishes the systematics of maximum superheating and undercooling based on classical nucleation theory and undercooling experiments. Molecular dynamics simulations of undercooling and superheating (Sec. III) were conducted to validate the empirical superheating-undercooling systematics at the level of interatomic interactions. Section IV presents the superheating results from dynamic melting experiments and their comparison to the superheating-undercooling systematics.

\section{SYSTEMATICS OF MAXIMUM UNDERCOOLING AND SUPERHEATING}

To study the maximum undercooling and superheating, we adopt classical theory of homogeneous nucleation. Heterogeneous nucleation theory is difficult to implement because it requires a detailed description of heterogeneous nucleation sites, and heterogeneous nucleation can be experimentally circumvented. ${ }^{1,2}$ Homogeneous nucleation theory supplies an upper bound to the maximum undercooling and superheating. For homogeneous nucleation of crystals from undercooled liquids (e.g., liquid metals), the time required for nuclei growth is much less than that for nucleation ${ }^{22}$; thus only the nucleation aspects are of interest. Indeed, a catastrophic increase in nucleation rate near the maximum undercooling (superheating) dominates the process of creating or breaking the long-range order. Various treatments of nucleation $^{1,23-27}$ share a common form for the steady-state nucleation rate $I$ (per unit volume):

$$
I=M(m, T) \exp \left\{-\frac{\Delta G_{c}}{k T} g(\phi)\right\},
$$

where $M$ is a function of material properties $(m)$ and temperature $(T) . \Delta G_{c}$ is the critical Gibbs free energy for nucleation, $k$ Boltzmann's constant, and $g(\phi)$ a geometrical factor depending on the wetting angle $\phi$ of a heterogeneous nucleant. For homogeneous nucleation, $g(\phi)=1$, the case assumed in the following discussions. Consider a spherical liq- uid nucleus of critical radius within a superheated crystal lattice (and similarly for nucleation of crystal within an undercooled liquid); the critical nucleation energy ${ }^{1,23} \Delta G_{c}$ $=16 \pi \gamma_{s l}^{3} /\left(3 \Delta G_{s l}^{2}\right)$ where $\gamma_{s l}$ is the solid-liquid interfacial energy and $\Delta G_{s l}$ the Gibbs free energy difference per unit volume between the solid and liquid state, approximated as $\Delta H_{m}\left(T-T_{m}\right) / T_{m}$ where $\Delta H_{m}$ is heat of fusion and $T_{m}$ melting temperature (assuming heat capacities of liquid and solid are approximately equal; see Refs. 1,17, and 23). The temperature dependence of the $M$ term in $I$ is negligible compared to that of the exponential $\Delta G_{c}$ term; thus $M$ can be regarded as a constant ${ }^{1,25,28} I_{0}$. We define the energy barrier for nucleation, $\beta$, as a dimensionless quantity,

$$
\beta\left(\gamma_{s l}, \Delta H_{m}, T_{m}\right)=\frac{16 \pi \gamma_{s l}^{3}}{3 \Delta H_{m}^{2} k T_{m}},
$$

and introduce the reduced temperature $\theta=T / T_{m}$. Thus $I$ $=I_{0} f(\beta, \theta)$ with

$$
f(\beta, \theta)=\exp \left\{-\frac{\beta}{\theta(\theta-1)^{2}}\right\} .
$$

The prefactor $I_{0}$ can be obtained experimentally or theoretically. This functional form applies to both melting and freezing cases. Nucleation is essentially controlled by $f(\beta, \theta)$, i.e., by the dimensionless energy barrier $\beta$ at given temperature. The form of $f(\beta, \theta)$ is simple but it does reflect the fundamental physics of nucleation. During superheating ( $\theta$ $>1$ ) of solids, $f$ (i.e., normalized nucleation rate) increases with temperature monotonically, as the mobility of atoms and the chemical driving force for melt nucleation both increase with $T$. On the other hand, during undercooling of liquids $(0<\theta<1)$, the thermodynamic driving force induced by undercooling is partly offset by the decrease in mobility; thus we have a maximum for $f$ at $\theta=1 / 3$. Although diffusion is not explicitly included in $f$, it is accounted for by the functional form of $f$.

Note that $f(\beta, \theta), \beta$, and $\theta$ are all dimensionless, allowing direct and convenient comparison of these quantities for different materials. $\beta$ is characteristic of a particular material, depending on $\gamma_{s l}, \Delta H_{m}$, and $T_{m}$. To estimate the value of $\beta$, we start with a hard sphere system (HSS) due to its simplicity and the availability of high-quality theoretical results. Consider hard spheres of diameter $\sigma$. The solid-liquid interfacial energy $\gamma_{s l}^{H S S}$ for a hard-sphere system has been shown to be $\mathrm{e}^{29} 0.61 \mathrm{kT} / \sigma^{2}$. The heat of fusion ${ }^{30}$ for a HSS, $\Delta H_{m}^{H S S}=1.16 k T$. Thus, $\beta \sim 0.77$ for a hard-sphere system. We may expect that for a real system the value of $\beta$ would be of similar magnitude. For a real system, $\gamma_{s l} \sim 0.1 \mathrm{~J} / \mathrm{m}^{2}$, $T_{m} \sim 10^{3} \mathrm{~K}$, and $\Delta H_{m} \sim 10^{9} \mathrm{~J} / \mathrm{m}^{3}$ yields $\beta \sim 1.2$. Undercooling experiments with homogeneous nucleation have been well documented in the literature (e.g., for elements ${ }^{1,27}$ ). The elements studied include transition metals, simple metals, and semiconductors in the third to sixth rows of the periodic table. Elements are simpler than compounds, yet their physical properties vary dramatically due to the variations in electronic structure. They show a range of properties relevant to 


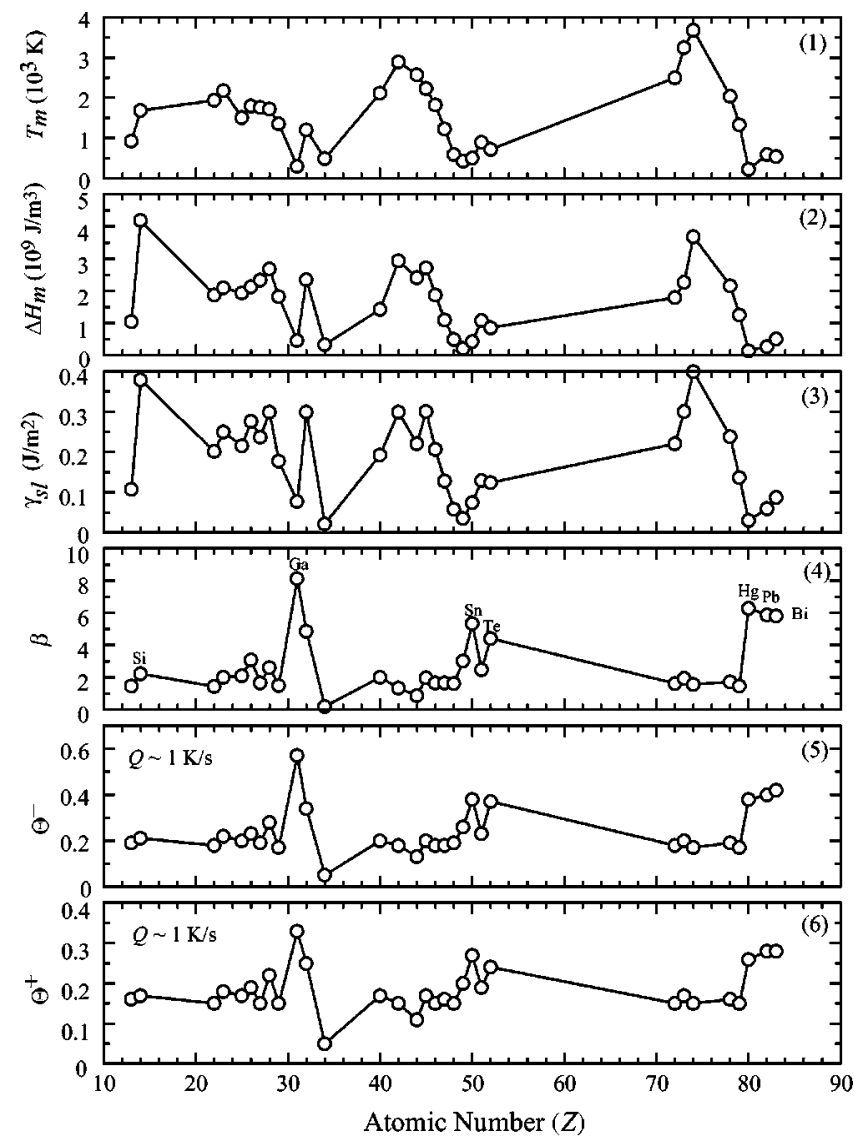

FIG. 1. Atomic number $(Z)$ vs melting point $\left(T_{m}\right.$, indexed as 1$)$, heat of fusion $\left(\Delta H_{m}, 2\right)$, solid-liquid interfacial energy $\left(\gamma_{s l}, 3\right)$, normalized energy barrier for nucleation $(\beta, 4)$, experimental undercooling $\left(\Theta^{-}, 5\right)$, and calculated superheating $\left(\Theta^{+}, 6\right)$ at $Q$ $\sim 1 \mathrm{~K} / \mathrm{s}$. Values for $T_{m}, \Delta H_{m}, \gamma_{s l}$ and $\Theta^{-}$are from the literature (Refs. 1 and 27).

more complex materials. Given $T_{m}, \Delta H_{m}$, and $\gamma_{s l}$ (Fig. 1), values of $\beta$ for these elements are readily calculated as shown in Figs. 1 and 2. Transition metals (group IVB-IIB) have relatively similar values of $\beta$ with an average close to 2 , except for $\mathrm{Hg}(\beta=6.30)$. Group IIA-VIA elements have significantly higher values of $\beta$, with a maximum at $\mathrm{Ga}(\beta$ $=8.15)$ except for $\mathrm{Al}(\beta=1.47)$ and Se which has the lowest $\beta=0.20$. These observations reflect the periodic variations in electronic structure in a general sense.

We calculate the cross-correlation coefficients among $T_{m}$, $\Delta H_{m}, \gamma_{s l}$, and $\beta$ (indexed as $1,2,3$, and 4 , respectively, in Fig. 1; also see Table I). As $R_{i j}=0.81-0.97(i, j=1,2,3)$, $T_{m}, \Delta H_{m}$, and $\gamma_{s l}$ vary with atomic number $Z$ in a similar manner. In particular, $R_{23}=0.97$ for $\gamma_{s l}$ and $\Delta H_{m}$. This indicates that compared to $T_{m}, \Delta H_{m}$ is a better indicator of $\gamma_{s l}$, which is by itself difficult to measure. $R_{i j} \sim 1$ also implies that these three parameters can be attributed to the same physical quantities such as binding energy or its closely related parameter, the heat of vaporization. $\gamma_{s l}$ increases with $T_{m}$ and $\Delta H_{m}$. Thus, although $\beta$ is sensitive to $\gamma_{s l}$, the variation in $\beta$ induced by that in $\gamma_{s l}$ could be offset by those in $T_{m}$ and $\Delta H_{m}$. To first order, $\left(\gamma_{s l} V^{2 / 3} N_{A}^{1 / 3}\right) /\left(\Delta H_{m} V\right) \sim$ const for a variety of elements and compounds, ${ }^{1,2}$ where $V$ is molar

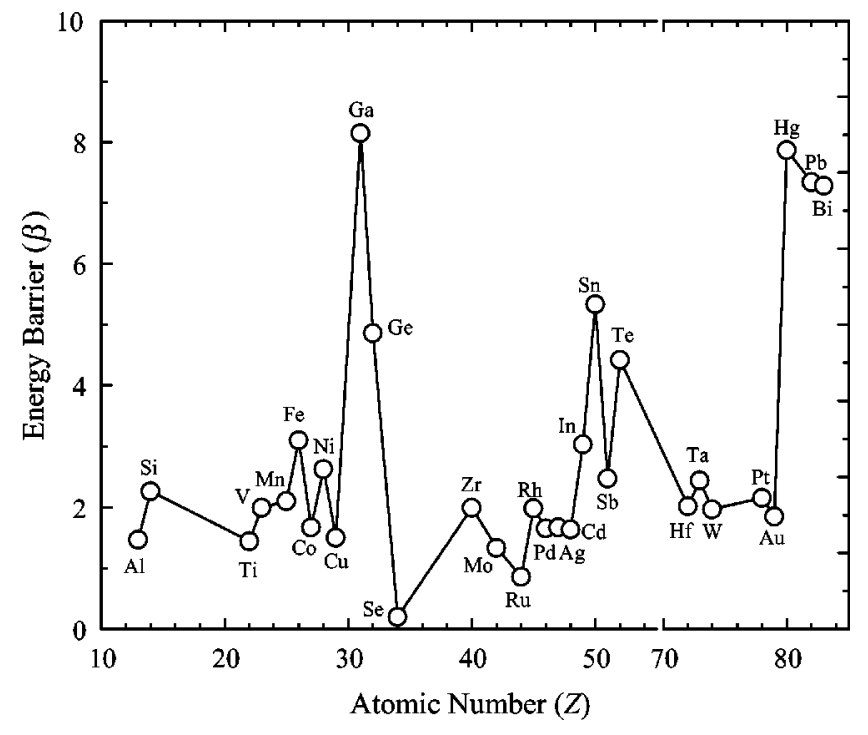

FIG. 2. Normalized energy barrier $\beta$ vs atomic number $Z$.

volume and $N_{A}$ Avogadro's number. Thus $\beta \propto \Delta S_{m}$ from its definition. As the entropy of fusion (per mole atoms) $\Delta S_{m}$ $\sim R, \beta$ should vary in a narrow range for different materials. The same arguments can be made with $\gamma_{s l} \propto \Delta H_{m} / a^{2}$ where $a^{2}$ is the effective atomic surface area. ${ }^{25}$ We also expect that $\beta$ for most materials should vary only slightly as pressure is increased (e.g., to megabar pressure under shock compression) because $\Delta S_{m}$ is given closely by $R \ln 2$ at high pressures. $^{31}$ Moreover, molecular dynamics simulations of superheating and undercooling of $\mathrm{Al}$ between 0 and $100 \mathrm{GPa}$ exhibit a weak pressure dependence in $\beta$ (see Sec. III). We also note that $\beta$ poorly correlates with $T_{m}, \Delta H_{m}$, and $\gamma_{s l}$ $\left(R_{i j}=-0.57,-0.46\right.$, and -0.38 , respectively; see Table I).

Having established the systematics of $\beta$, we next explore the relationship of $T_{m}, \Delta H_{m}, \gamma_{s l}$, and $\beta$ to the maximum undercooling and superheating, denoted as $\theta_{c}=T_{c} / T_{m}$ where $T_{c}$ is either the highest temperature achieved in the superheated solid or the lowest temperature in the undercooled liquid. The maximum undercooling is also denoted by $\Theta^{-}$ $=1-\theta_{c}$ and superheating as $\Theta^{+}=\theta_{c}-1$. The cooling rate $Q \sim 1 \mathrm{~K} / \mathrm{s}$ is typical for conventional undercooling experiments. We represent this rate by a subscript $s$ and the rate $10^{12} \mathrm{~K} / \mathrm{s}$ by a subscript $n s$. Experimental values of $\Theta_{s}^{-}$for elements and calculated $\Theta_{s}^{+}$(discussed next) are plotted in Fig. 1 (indexed as 5 and 6 , respectively). $\Theta_{s}^{-}$and $\Theta_{s}^{+}$increase with $\beta$, with correlations $R_{45}=0.98$ and $R_{46}=0.96$ (Table I). However, $\Theta_{s}^{-}\left(\Theta_{s}^{+}\right)$correlates poorly with any of $\gamma_{s l}, T_{m}$, and $\Delta H_{m}$. This is possible because the nucleation rate $I$ depends on the combination of these parameters. Thus,

TABLE I. Cross-correlation coefficient $R_{i j} . T_{m}, \Delta H_{m}, \gamma_{s l}, \beta$, $\Theta^{-}$, and $\Theta^{+}$are indexed as $1,2,3,4,5$, and 6 , respectively (also see Fig. 1).

\begin{tabular}{ccccccccc}
\hline \hline$R_{12}$ & $R_{13}$ & $R_{23}$ & $R_{14}$ & $R_{24}$ & $R_{34}$ & $R_{45}$ & $R_{46}$ & $R_{56}$ \\
\hline 0.81 & 0.84 & 0.97 & -0.57 & -0.46 & -0.38 & 0.98 & 0.96 & 0.98 \\
\hline \hline
\end{tabular}


$\beta$, involving $\gamma_{s l}, T_{m}$, and $\Delta H_{m}$, better captures the physics of the nucleation and adequately represents variations in the maximum undercooling and superheating. It also goes against conventional wisdom that materials with low $T_{m}$ should demonstrate less maximum superheating or undercooling. Counterexamples include elements like $\mathrm{Ga}$ and $\mathrm{Hg}$, which have low $T_{m}$ (303 and $234 \mathrm{~K}$, respectively) but high values of $\Theta_{s}^{-}(0.57$ and 0.38$)$. Indeed, compared with $\gamma_{s l}$ and $\Delta H_{m}, T_{m}$ is better anticorrelated with $\Theta^{-}$and $\beta$ : most low$T_{m}$ materials surprisingly have high values of $\beta$ and $\Theta_{s}^{-}$ (Fig. 1). But this should not be overinterpreted, as $R_{24}$ $=-0.57$, which is significantly less negative than -1 . The best parameter for studying nucleation is $\beta$.

In contrast with the large body of undercooling data with $Q \sim 1 \mathrm{~K} / \mathrm{s}$, data are rarely available with appreciable superheating at heating rates of $\sim 1 \mathrm{~K} / \mathrm{s}$ as heterogeneous nucleation dominates at these low rates. It would be of great interest to predict the maximum undercooling and superheating under various cooling and heating rates, e.g., $Q \sim 10^{12} \mathrm{~K} / \mathrm{s}$. Given the systematics of $\beta$ and experimental values of $\Theta_{s}^{-}$, we next develop a scheme to predict the maximum undercooling and superheating under different cooling and heating rates.

For steady-state homogeneous nucleation of crystal in liquid (or melt in crystal), the probability ${ }^{1} x$ for a given amount of parent phase of volume $v$ containing no new phase under certain cooling (or heating) rate $Q$ is

$$
x=\exp \left\{ \pm \frac{v T_{m} I_{0}}{Q} \int_{\theta_{c}}^{1} f(\beta, \theta) d \theta\right\},
$$

where + refers to superheating and - to undercooling. The parameters for undercooling experiments at $Q \sim 1 \mathrm{~K} / \mathrm{s}$, such as $\gamma_{s l}, \Delta H_{m}, T_{m}$ (thus $\beta$ ), and $v$, can be regarded as equal to those for superheating and undercooling at different heating and cooling rates. By assuming that $x$ and $I_{0}$ is approximately equal for the undercooling and superheating cases, the maximum superheating and undercooling under any $Q$ can be calculated from the experimental value of $\Theta_{s}^{-}$. For example, the maximum superheating $\Theta^{+}$at any heating rate $Q$ can be found from

$$
\frac{1}{Q} \int_{1}^{\left(1+\Theta^{+}\right)} f(\beta, \theta) d \theta=\frac{1}{Q_{0}} \int_{\left(1-\Theta_{s}^{-}\right)}^{1} f(\beta, \theta) d \theta,
$$

where $Q_{0}=1 \mathrm{~K} / \mathrm{s}$. Similarly, the maximum undercooling $\Theta^{-}$under various cooling rates $Q$ can be readily calculated. Thus, for a given material with $\beta$ and $\Theta_{s}^{-}$, we can predict the maximum superheating and undercooling at any heating and cooling rate. Figure 3 shows the experimental value of $\theta_{c}$ at the maximum undercooling $\left(\theta_{c}=1-\Theta_{s}^{-}\right.$, circles $)$and the calculated $\theta_{c}$ at the maximum superheating $\left(\theta_{c}=1\right.$ $+\Theta_{s}^{+}$, diamonds) at $Q=1 \mathrm{~K} / \mathrm{s}$, for elements. Note that heating (cooling) rates such as 1 and $10^{12} \mathrm{~K} / \mathrm{s}$ should be regarded as adequately representative, because a factor-of $-10^{2}$ change in $Q$ would yield negligible changes in $\theta_{c}$ (except for high- $\beta$ elements such as Ga upon undercooling), due to the functional form of $f(\beta, \theta)$. When calculating maximum undercooling at high cooling rates, $\Theta^{-}$might be 1 (essentially $2 / 3$

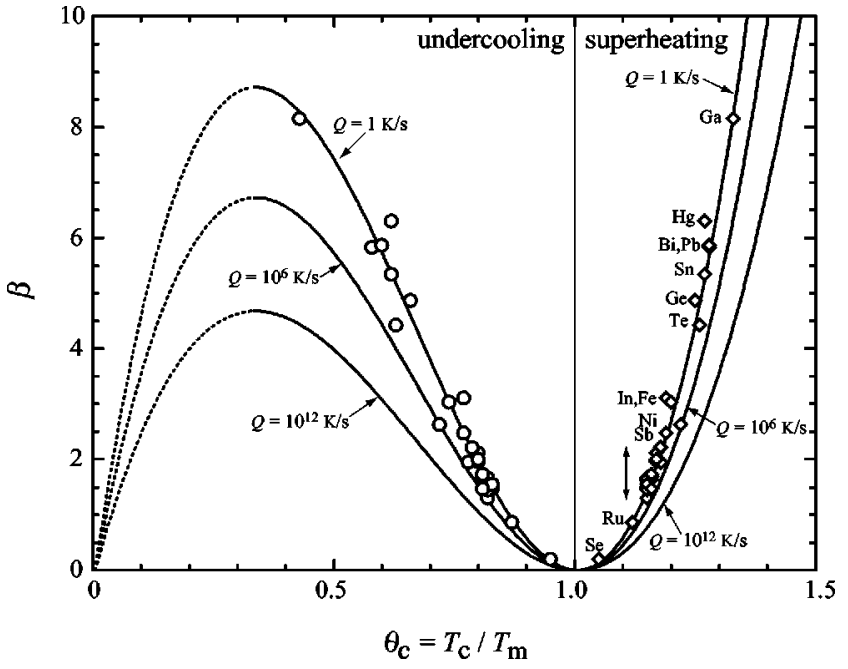

FIG. 3. The systematics of maximum superheating and undercooling for elements: $\beta=\left(A_{0}-b \log _{10} Q\right) \theta_{c}\left(1-\theta_{c}\right)^{2}$. Circles are experimental value of undercooling at cooling rate $Q \sim 1 \mathrm{~K} / \mathrm{s}$, and diamonds are calculated superheating at $Q \sim 1 \mathrm{~K} / \mathrm{s}$. Solid and dotted curves are plots with $Q=1,10^{6}$, and $10^{12} \mathrm{~K} / \mathrm{s}$, respectively. Dotted curves denote the undercooling portions for $\theta_{c}=0-\frac{1}{3}$. The elements within the double-headed arrow are $\mathrm{Ti}, \mathrm{Al}, \mathrm{Au}, \mathrm{Cu}, \mathrm{Hf}$, $\mathrm{Cd}, \mathrm{Pd}, \mathrm{Ag}, \mathrm{Co}, \mathrm{Pt}, \mathrm{Ta}, \mathrm{Rh}, \mathrm{Zr}, \mathrm{Mn}, \mathrm{Si}, \mathrm{Sb}, \mathrm{Ni}, \mathrm{In}$, and $\mathrm{Fe}$ in $\beta$-increasing order. The maximum of $\beta$ occurs at $\theta_{c}=1 / 3$ for each $Q$.

as shown next). For instance, $\Theta^{-}=1$ for $\mathrm{Ga}$ at $Q=10^{6} \mathrm{~K} / \mathrm{s}$ and for $\mathrm{Bi}, \mathrm{Ga}, \mathrm{Pb}, \mathrm{Sn}$, and $\mathrm{Te}$ at $Q=10^{12} \mathrm{~K} / \mathrm{s} . \Theta^{-}=1$ indicates that under such high cooling rates, these liquids would not solidify as crystals.

The relationship between the material property $\beta$, heating (cooling) rate $Q$, and maximum superheating (undercooling) $\theta_{c}$ is obtained empirically as

$$
\beta=A(Q) \theta_{c}\left(\theta_{c}-1\right)^{2},
$$

where $A$ is a fitting constant depending on $Q$. Fitting the undercooling and superheating cases independently, similar values of $A$ were obtained, indicating that this functional form describes both superheating and undercooling with a unique $A(Q)$. Such fittings to both superheating and undercooling yield $A(Q)=59.4,45.4$, and 31.4 for $1,10^{6}$, and $10^{12} \mathrm{~K} / \mathrm{s}$, respectively (Fig. 3). While a precise physical interpretation is not clear, $A$ may be regarded as reflecting a relative time scale characteristic of nucleation at different heating (cooling) rates. The fitting process was repeated at different $Q$, and $A$ was found to vary linearly with $\log _{10} Q$. Thus we can rewrite Eq. (6) as

$$
\beta=\left(A_{0}-b \log _{10} Q\right) \theta_{c}\left(\theta_{c}-1\right)^{2},
$$

where $A_{0}=59.4, b=2.33$, and $Q$ is normalized by $Q_{0}$ $=1 \mathrm{~K} / \mathrm{s}$. Equation (7) is referred to as the $\theta_{c}-\beta-Q$ systematics for the maximum superheating and undercooling.

There are some important features in the $\theta_{c}-\beta-Q$ systematics [Eq. (7) and Fig. 3]. The functional form implies that $f\left(\beta, \theta_{c}\right)=\exp [-A(Q)]$ and can be regarded as a constant for a given $Q$. The nucleation rate near $\theta_{c}$ dominates the nucle- 
TABLE II. Comparison of the calculated maximum superheating from Ref. 19 (assuming $I=1 \mathrm{~cm}^{-3} \mathrm{~s}^{-1}$ ) and this work at $1 \mathrm{~K} / \mathrm{s}$.

\begin{tabular}{lccccccccccccc}
\hline \hline & $\mathrm{Sn}$ & $\mathrm{Pb}$ & $\mathrm{Sb}$ & $\mathrm{Al}$ & $\mathrm{Ag}$ & $\mathrm{Au}$ & $\mathrm{Cu}$ & $\mathrm{Mn}$ & $\mathrm{Ni}$ & $\mathrm{Co}$ & $\mathrm{Fe}$ & $\mathrm{Pd}$ & $\mathrm{Pt}$ \\
\hline Ref. 19 & 0.279 & 0.291 & 0.192 & 0.208 & 0.179 & 0.184 & 0.174 & 0.177 & 0.212 & 0.184 & 0.212 & 0.183 & 0.185 \\
This work & 0.265 & 0.280 & 0.188 & 0.158 & 0.155 & 0.145 & 0.150 & 0.170 & 0.216 & 0.154 & 0.190 & 0.154 & 0.156 \\
\hline \hline
\end{tabular}

ation process during undercooling and superheating. As shown in Fig. 3, there is an asymmetry of the maximum superheating and undercooling $\theta_{c}$ (relative to $\theta_{c}=1$ ) at the same $Q$; i.e., for a material with certain $\beta$, it can achieve a larger amount of maximum undercooling than maximum superheating. This is reasonable because the temperature and hence atomic mobility are higher for superheating. The increase of maximum superheating induced by increasing $Q$ is less pronounced than the undercooling case; e.g., for $\mathrm{Al}$, $\Theta_{n s}^{+}-\Theta_{s}^{+}=0.07$ while $\Theta_{n s}^{-}-\Theta_{s}^{-}=0.11$. Increasing $Q$ does not significantly increase the maximum superheating: a change in $Q$ from 1 to $10^{12} \mathrm{~K} / \mathrm{s}$ yields an increase of $<10 \%$ in $\theta_{c}$. The maximum $\Theta^{+}<0.45$ even at $Q=10^{12} \mathrm{~K} / \mathrm{s}$. But increasing the heating rate does serve as an important approach to achieving superheating experimentally (Sec. IV). There is a maximum of $\beta$ at $\theta_{c}=1 / 3$ for any cooling ratee.g., $\beta_{\text {max }}=8.72,6.73$, and 4.68 at $1,10^{6}$, and $10^{12} \mathrm{~K} / \mathrm{s}$, respectively. For any given cooling rate $Q$, materials with $\beta>\beta_{\text {max }}=\frac{4}{27}\left(A_{0}-b \log _{10} Q\right)$ will not crystallize, and liquids may persist or become glassy, depending on whether the glass transformation temperature is higher or lower than $\theta_{c} \cdot{ }^{32}$ This occurs because of the trade-off between the increasing thermodynamic driving force for crystallization and the decreasing atomic mobility as the temperature falls. The largest possible undercooling for any material at all cooling rates is $\Theta^{-}=2 / 3$ which is also implied by $f(\beta, \theta)$ [Eq. (3)]. This result has been obtained using different approaches. ${ }^{22,32}$ For the data we have collected, the largest $\Theta_{s}^{-}$exhibited is 0.57 for $\mathrm{Ga}$. There could exist an uninvestigated material with $\beta=8.72$ such that $\Theta_{s}^{-}=0.67$. An increased cooling rate may induce $\Theta^{-}=2 / 3$; e.g., such undercooling may be achieved for $\mathrm{Ga}$ at $Q \sim 10^{2} \mathrm{~K} / \mathrm{s}$.

Previously, theories involving catastrophe in entropy, ${ }^{17}$ rigidity, and volume ${ }^{18}$ predict a wide range ${ }^{19}\left(\Theta^{+}=0.3-2.0\right)$ of maximum superheating. Other efforts ${ }^{19,20}$ to describe superheating assumed $I=1 \mathrm{~cm}^{-3} \mathrm{~s}^{-1}$ or a critical volume. Generally, the variation of heating rates was not considered. We have established the $\theta_{c}-\beta-Q$ systematics [Eq. (7) and Fig. 3] based on undercooling experiments and homogeneous nucleation theory, and have incorporated the effect of altering the heating or cooling rate. The maximum superheating at $Q=1 \mathrm{~K} / \mathrm{s}$ predicted from Eq. (7) is slightly lower than that from Ref. 19 (Table II). $I=1 \mathrm{~cm}^{-3} \mathrm{~s}^{-1}$ is a reasonable first-order value at low $Q$. A direct application of the systematics is that, given measured maximum undercooling and superheating, we can determine $\beta$ and predict the maximum superheating and undercooling at other heating and cooling rates. Knowledge of $\beta$ also allows an evaluation of $\gamma_{s l}$ given $\Delta H_{m}$ and $T_{m}$ which are in general available. Systematic undercooling experiments have been conducted on alkali halides. ${ }^{25}$ The interfacial energy and maximum undercooling and superheating at typical cooling and heating rates were predicted (Table III). For example, the maximum superheating achievable for $\mathrm{CsBr}$ at $10^{12} \mathrm{~K} / \mathrm{s}$ is 0.20 . Similarly, given superheating measurements on silicates, ${ }^{2}$ we can predict $\gamma_{s l}$ and the maximum undercooling and superheating under various $Q$ (Table III).

\section{MOLECULAR DYNAMICS SIMULATIONS OF MAXIMUM SUPERHEATING AND UNDERCOOLING}

The systematics of the maximum superheating and undercooling [Eq. (7)] established in Sec. II are empirical in nature. Next we investigate the maximum superheating and undercooling achieved in molecular dynamics simulations (with $Q \sim 10^{12} \mathrm{~K} / \mathrm{s}$ ) and their relationship to the systematics.

Simulations were made of a supercell with 3D periodic boundaries subjected to incremental heating at constant pressure $P$, e.g., with isothermal-isobaric statistical ensemble $N-P-T$ where $N$ is the total number of atoms in the supercell. The temperature was increased until the crystal was observed to have melted; then the system was cooled incrementally until it refroze. Hysteresis was observed in association with

TABLE III. Undercooling and superheating parameters of alkali halides and silicates based on the experiments and the maximum superheating-undercooling systematics [Eq. (7)].

\begin{tabular}{|c|c|c|c|c|c|c|c|c|}
\hline & $\begin{array}{l}T_{m} \\
(\mathrm{~K})\end{array}$ & $\begin{array}{c}\gamma_{s l} \\
\left(\mathrm{~J} / \mathrm{m}^{2}\right)\end{array}$ & $\begin{array}{c}\Delta H_{m} \\
(\mathrm{~kJ} / \mathrm{mol})\end{array}$ & $\beta$ & $\Theta_{s}^{-}$ & $\Theta_{n s}^{-}$ & $\Theta_{s}^{+}$ & $\Theta_{n s}^{+}$ \\
\hline \multicolumn{9}{|c|}{ Alkali halides ${ }^{a}$} \\
\hline $\mathrm{CsBr}$ & 909 & 0.066 & 23.58 & 1.56 & 0.18 & 0.26 & 0.15 & 0.20 \\
\hline $\mathrm{CsCl}$ & 919 & 0.051 & 15.06 & 1.41 & 0.17 & 0.24 & 0.14 & 0.19 \\
\hline $\mathrm{CsF}$ & 955 & 0.046 & 10.25 & 1.27 & 0.16 & 0.23 & 0.14 & 0.18 \\
\hline CsI & 894 & 0.069 & 24.66 & 2.40 & 0.23 & 0.34 & 0.19 & 0.25 \\
\hline $\mathrm{KBr}$ & 1003 & 0.063 & 20.92 & 1.27 & 0.16 & 0.23 & 0.14 & 0.18 \\
\hline $\mathrm{KCl}$ & 1049 & 0.082 & 26.82 & 1.27 & 0.16 & 0.23 & 0.14 & 0.18 \\
\hline KI & 959 & 0.047 & 17.15 & 1.27 & 0.16 & 0.23 & 0.14 & 0.18 \\
\hline $\mathrm{LiBr}$ & 820 & 0.046 & 12.13 & 0.63 & 0.11 & 0.15 & 0.10 & 0.13 \\
\hline $\mathrm{LiCl}$ & 887 & 0.086 & 13.39 & 2.05 & 0.21 & 0.31 & 0.17 & 0.23 \\
\hline $\mathrm{LiF}$ & 1115 & 0.124 & 9.87 & 2.05 & 0.21 & 0.31 & 0.17 & 0.23 \\
\hline $\mathrm{NaBr}$ & 1028 & 0.088 & 25.69 & 1.27 & 0.16 & 0.23 & 0.14 & 0.18 \\
\hline $\mathrm{NaCl}$ & 1074 & 0.112 & 30.21 & 1.27 & 0.16 & 0.23 & 0.14 & 0.18 \\
\hline $\mathrm{NaF}$ & 1261 & 0.206 & 29.29 & 2.22 & 0.22 & 0.32 & 0.18 & 0.24 \\
\hline $\mathrm{RbCl}$ & 988 & 0.057 & 18.41 & 1.27 & 0.16 & 0.23 & 0.14 & 0.18 \\
\hline \multicolumn{9}{|c|}{ Silicates ${ }^{b}$} \\
\hline $\mathrm{NaAlSi}_{3} \mathrm{O}_{8}$ & 1371 & 0.088 & 63.00 & 1.52 & 0.18 & 0.25 & 0.15 & 0.20 \\
\hline $\mathrm{SiO}_{2}$ & 1700 & 0.106 & 9.40 & 5.01 & 0.37 & 1.00 & 0.26 & 0.34 \\
\hline
\end{tabular}

${ }^{\mathrm{a} U n d e r c o o l i n g ~ d a t a ~}\left(\Theta_{s}^{-}\right)$are from Ref. 25.

${ }^{\mathrm{b}}$ Superheating data $\left(\Theta_{s}^{+}\right)$for albeit and quartz are from Ref. 2. 
heating or cooling at these finite rates. The melting temperature determined by heating the solid phase (single-phase melting temperature $T_{1, m}$ ) was higher than the equilibrium $T_{m}$, demonstrating a superheating component in the hysteresis. Conversely, the crystallization temperature by cooling the liquid phase (single-phase crystallization temperature $T_{1, c}$ ) was lower than $T_{m}$. We refer to this procedure as single-phase simulation. To quantify the degree of superheating and undercooling, we need to find the equilibrium melting temperature $T_{m}$. Given a specific force field (FF) describing interatomic interactions, $T_{m}$ can be determined by such techniques as thermodynamic integration of free energy ${ }^{33}$ and solid-liquid coexisting phase simulations. ${ }^{10}$ The latter technique is a natural choice for our purpose. Assuming that superheating and undercooling reflect the nucleation process, they can be circumvented by constructing a solidliquid coexisting (two-phase) system with a planar solidliquid interface. As both phases are present along with a boundary region, nucleation-related superheating or undercooling is avoided. ${ }^{10}$ We refer to simulations conducted on such a two-phase system as two-phase simulations. Thus we can determine the equilibrium melting temperature from the two-phase simulation, $T_{2, m}$, for the specific system with an assigned FF. In this way, we can quantify the degree of maximum superheating $\left(\Theta_{m d}^{+}=T_{1, m} / T_{2, m}-1\right)$ and undercooling $\left(\Theta_{m d}^{-}=1-T_{1, c} / T_{2, m}\right)$ in MD simulations. In this work, we conducted single- and two-phase simulations of the melting and refreezing of close-packed metals. These simulations along with previous work ${ }^{13,34,35}$ allow us to systematically examine superheating and undercooling behavior for elements and compounds described with different potentials against the maximum superheating-undercooling systematics developed. The pressure effect on superheating is also addressed.

To simulate the close-packed (fcc and hcp) metals, we adopt Sutton-Chen (SC) many-body potential ${ }^{36}$ with quantum corrections (qSC). ${ }^{37}$ For SC FF's, the total potential energy of the system, $U_{t o t}$, is

$$
U_{t o t}=\sum_{i} \epsilon\left[\sum_{j \neq i} \frac{1}{2} V\left(r_{i j}\right)-c \rho_{i}^{1 / 2}\right] .
$$

The pair potential

$$
V\left(r_{i j}\right)=\left(\frac{a}{r_{i j}}\right)^{n}
$$

accounts for the repulsion between the atoms $i$ and $j$ where $r_{i j}$ is the separation between them. The many-body cohesion is accounted for with the local electron density on atom $i$ :

$$
\rho_{i}=\sum_{j \neq i}\left(\frac{a}{r_{i j}}\right)^{m}
$$

The parameters $(a, \epsilon, c, m, n)$ were obtained by optimization to best-fit empirical values including lattice parameter, cohesive energy, elastic constants, etc. The melting point was not included. The quantum effect (e.g., zero-point vibrational
TABLE IV. The qSC FF for fcc and hcp metals (Ref. 37).

\begin{tabular}{lccccc}
\hline \hline & $a(\AA)$ & $\epsilon\left(10^{-2} \mathrm{eV}\right)$ & $c$ & $m$ & $n$ \\
\hline $\mathrm{Be}$ & 3.18863 & 0.18207 & 222.34769 & 6 & 13 \\
$\mathrm{Al}$ & 4.03230 & 0.90144 & 54.97923 & 5 & 9 \\
$\mathrm{Ni}$ & 3.51570 & 0.73767 & 84.74500 & 5 & 10 \\
$\mathrm{Cu}$ & 3.60300 & 0.57921 & 84.84300 & 5 & 10 \\
$\mathrm{Rh}$ & 3.79840 & 0.24612 & 305.49900 & 5 & 13 \\
$\mathrm{Pd}$ & 3.88130 & 0.32864 & 148.20500 & 6 & 12 \\
$\mathrm{Ag}$ & 4.06910 & 0.39450 & 96.52400 & 6 & 11 \\
$\mathrm{Ir}$ & 3.83440 & 0.37674 & 224.81500 & 6 & 13 \\
$\mathrm{Pt}$ & 3.91630 & 0.97894 & 71.33600 & 7 & 11 \\
$\mathrm{Au}$ & 4.06510 & 0.78052 & 53.58100 & 8 & 11 \\
$\mathrm{~Pb}$ & 4.94950 & 0.55772 & 45.88200 & 7 & 10 \\
\hline \hline
\end{tabular}

energy) was also included in the optimization. ${ }^{37}$ The parameters of such a qSC force field for close-packed metals are listed in Table IV.

Given the force field, we constructed a supercell of 864 atoms for each fcc metal and 1024 atoms for Be with 3D periodic boundaries. MD simulations were conducted with an $N-P-T$ ensemble using a Hoover thermostat ${ }^{38}$ and a Rahman-Parinello barostat. ${ }^{39}$ The system was subjected to incremental heating and cooling at a rate of $5 \mathrm{~K} / \mathrm{ps}$ (5 $\left.\times 10^{12} \mathrm{~K} / \mathrm{s}\right)$. At the end of each heating and cooling step, physical properties such as $P$, density, and $T$ were calculated statistically. Thus a plot of density versus $T$ at constant $P$ can be obtained from $N-P-T$ runs, and the temperatures at the first-order phase transitions (melting and freezing, $T_{1, m}$ and $T_{1, c}$ ) were readily obtained by inspection in the plot. Figure 4(a) is a typical example (Al) of the melting-refreezing hysteresis: the system undergoes superheating before melting and undercooling before refreezing. To find the equilibrium melting temperature for the system with the prescribed force fields, we constructed a two-phase system with solid and liquid models at a common temperature from single-phase simulations. For the two-phase system, we performed $N-P-T$ runs at different temperatures: if $T>T_{m}$, the solid portion in
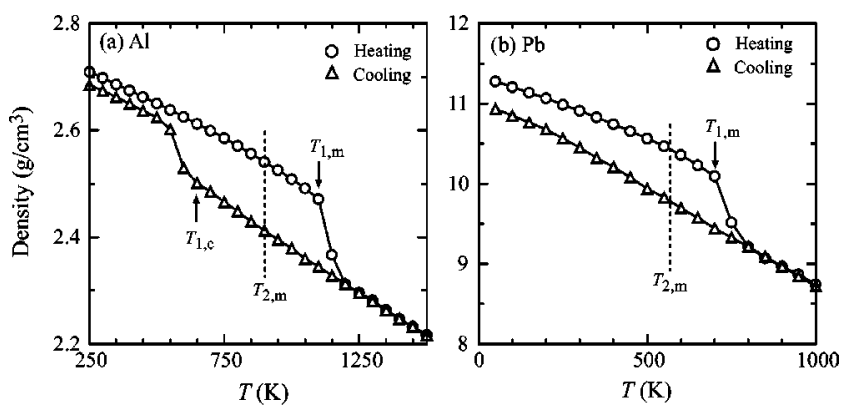

FIG. 4. Typical single- and two-phase molecular dynamics simulations of the melting and refreezing behavior: density vs $T$. A complete hysteresis of density forms during continuous heatingcooling process for $\mathrm{Al}(\mathrm{a})$, while for $\mathrm{Pb}(\mathrm{b})$ liquid eventually becomes glass upon undercooling. $T_{1, m}$ and $T_{1, c}$ are the single-phase melting and freezing temperature at the superheated and undercooled states, respectively. $T_{2, m}$ is the equilibrium melting temperature from the two-phase simulations. 
TABLE V. Single- and two-phase MD simulations of melting and refreezing of metals at $P=0$. Subscript 1 denotes single-phase simulation, 2 two-phase simulation, $m$ melting, $c$ crystallization, $e$ experiment, $m d$ molecular dynamics, and $n s 10^{12} \mathrm{~K} / \mathrm{s}$. $\Theta_{n s}^{-}$and $\Theta_{n s}^{+}$are calculated from Eq. (7).

\begin{tabular}{lcccccccccc}
\hline \hline & $\begin{array}{c}T_{1, m} \\
(\mathrm{~K})\end{array}$ & $\begin{array}{c}T_{1, c} \\
(\mathrm{~K})\end{array}$ & $\begin{array}{c}T_{2, m} \\
(\mathrm{~K})\end{array}$ & $\begin{array}{c}T_{e, m} \\
(\mathrm{~K})\end{array}$ & $\begin{array}{c}\Delta H_{m} \\
(\mathrm{~kJ} / \mathrm{mol})\end{array}$ & $\begin{array}{c}\gamma_{s l}^{\mathrm{a}} \\
\left(\mathrm{J} / \mathrm{m}^{2}\right)\end{array}$ & $\Theta_{m d}^{-}$ & $\Theta_{n s}^{-}$ & $\Theta_{m d}^{+}$ & $\Theta_{n s}^{+}$ \\
\hline $\mathrm{Be}$ & 1600 & 900 & 1350 & 1560 & 15.55 & 0.248 & 0.33 & - & 0.19 & - \\
$\mathrm{Al}$ & 1100 & 650 & 925 & 933 & 9.08 & 0.095 & 0.30 & 0.25 & 0.19 & 0.20 \\
$\mathrm{Ni}$ & 1700 & 1000 & 1375 & 1728 & 13.55 & 0.221 & 0.28 & 0.36 & 0.24 & 0.26 \\
$\mathrm{Cu}$ & 1350 & 750 & 1070 & 1356 & 10.80 & 0.151 & 0.30 & 0.24 & 0.21 & 0.19 \\
$\mathrm{Rh}$ & 2700 & 1600 & 2125 & 2239 & 25.56 & 0.365 & 0.25 & 0.30 & 0.27 & 0.23 \\
$\mathrm{Pd}$ & 1850 & 1000 & 1475 & 1825 & 16.28 & 0.216 & 0.32 & 0.27 & 0.25 & 0.21 \\
$\mathrm{Ag}$ & 1200 & 650 & 1000 & 1234 & 10.78 & 0.111 & 0.25 & 0.27 & 0.20 & 0.21 \\
$\mathrm{Ir}$ & 3400 & 1750 & 2740 & 2683 & 31.76 & 0.415 & 0.36 & - & 0.24 & - \\
$\mathrm{Pt}$ & 2450 & 1300 & 1925 & 2042 & 20.93 & 0.291 & 0.32 & 0.28 & 0.27 & 0.21 \\
$\mathrm{Au}$ & 1400 & 600 & 1075 & 1336 & 12.38 & 0.169 & 0.44 & 0.25 & 0.30 & 0.20 \\
$\mathrm{~Pb}$ & 700 & - & 575 & 601 & 5.93 & 0.045 & $\mathrm{vitri}$ & 1.00 & 0.22 & 0.37 \\
$\mathrm{Ta}{ }^{\mathrm{b}}$ & 3650 & - & 3150 & 3253 & 24.70 & 0.222 & - & 0.30 & 0.15 & 0.23 \\
\hline \hline
\end{tabular}

${ }^{\mathrm{a} C a l c u l a t e d ~ f r o m ~ t h e ~ s u p e r h e a t i n g-u n d e r c o o l i n g ~ s y s t e m a t i c s ~ a n d ~} \Theta_{m d}^{+}$.

${ }^{\mathrm{b}}$ Simulated with embedded-atom-method force field based on quantum mechanics calculations (Ref. 34).

the two-phase system melted and $T$ was reduced for the next run and vice versa. In this way the equilibrium melting temperature of the two-phase system $\left(T_{2, m}\right)$ was bracketed within a range of $25 \mathrm{~K}$.

From MD simulations, we obtained $T_{1, m}, T_{1, c}$, and $T_{2, m}$ at a given pressure; thus we can quantify the maximum superheating $\left(\Theta_{m d}^{+}\right)$and undercooling $\left(\Theta_{m d}^{-}\right)$achieved for a material with the specific force field. The maximum superheating and undercooling achieved at ambient pressure are summarized in Table $\mathrm{V} . \Theta_{m d}^{+}$and $\Theta_{m d}^{-}$vary in the range of $0.19-0.30$ and $0.25-0.44$ (except for $\mathrm{Pb}$ ), respectively. These values of $\Theta_{m d}^{+}$and $\Theta_{m d}^{-}$are comparable to the predictions of the superheating-undercooling systematics $\left(\Theta_{n s}^{+}\right.$and $\left.\Theta_{n s}^{-}\right)$at a similar heating (cooling) rate $\left(Q \sim 10^{12} \mathrm{~K} / \mathrm{s}\right)$ for the real systems. Interestingly, the MD simulations of undercooling in $\mathrm{Pb}$ in $\mathrm{MD}$ simulations predicted that the undercooled liquid eventually becomes glass rather than crystal [Fig. 4(b) or by calculating the radial distribution function]. This appears to be predicted by the systematics: at a cooling rate $\sim 10^{12} \mathrm{~K} / \mathrm{s}$ [Eqs. (7) and Fig. 3], a solid with $\beta>4.68$ [e.g., $\beta(\mathrm{Pb})=5.87]$ does not crystallize.

High-pressure melting-for example, melting under pressures comparable to shock wave loading-is of particular interest. Here we explore the pressure effect on superheating and undercooling. Single- and two-phase simulations were conducted on Al at $0,20,40,60,80$, and $100 \mathrm{GPa}$ (Table VI). $\Theta_{m d}^{+}$and $\Theta_{m d}^{-}$for $0 \leqslant P \leqslant 100 \mathrm{GPa}$ lie between $0.19-0.25$ and $0.30-0.48$, respectively. There is no obvious pressure dependence for the maximum superheating and undercooling in the case of Al. This seems to support the weak pressure dependence of $\beta$ as argued in the preceding section. Previously, the two-phase simulation technique has been employed to simulate the melting behavior of other materials described with different force fields. For example, the quantum-mechanics-based embedded-atom-method (EAM) force field was applied to $\mathrm{Ta}$ (bcc) and $\Theta_{m d}^{+}=0.15$ was obtained $^{34}$ (Table V). Simulations with an EAM force field for $\epsilon$-Fe (hcp) yielded $T_{1, m} \sim 8600 \mathrm{~K}, T_{2, m} \sim 7100 \mathrm{~K}$, and $\Theta_{m d}^{+} \sim 0.21,{ }^{35}$ close to the prediction $\Theta_{n s}^{+}=0.23$ if $\beta=3.11$ is assumed. For silica's high-pressure phase stishovite with a Morse-stretch-charge-equilibrium $\mathrm{FF}, \quad \Theta_{m d}^{+}=0.28$ was achieved at $120 \mathrm{GPa}{ }^{13}$

The comparison above between MD simulations and the predictions of the superheating-undercooling systematics assumed that the force fields utilized in MD simulations accurately describe real systems; this is not necessarily the case. The equilibrium melting temperature from MD simulations $\left(T_{2, m}\right)$ deviates from the experimental counterpart $\left(T_{e, m}\right)$ at ambient pressure for some metals (Table V). As the only exception, the undercooled $\mathrm{Pb}$ liquid does not refreeze in MD simulations while $\Theta_{m d}^{+}(\mathrm{Pb})=0.22$ indicates that the liquid should freeze with $\Theta_{m d}^{-}=0.29$ according to the systematics [Eq. (7)]. Such discrepancies imply that the force fields we employed do not necessarily represent all the behavior of real systems. To check whether MD simulations are consistent with the superheating-undercooling systematics, it is not appropriate to compare MD simulations directly with real systems, although they were certainly in reasonable accord. It is not our purpose in this work to develop or improve a force field and check its accuracy. Instead, we regard the

TABLE VI. Single- and two-phase MD simulations of melting and refreezing of $\mathrm{Al}$ at high pressures.

\begin{tabular}{lccccc}
\hline \hline$P(\mathrm{GPa})$ & $T_{1, m}(\mathrm{~K})$ & $T_{1, c}(\mathrm{~K})$ & $T_{2, m}(\mathrm{~K})$ & $\Theta_{m d}^{+}$ & $\Theta_{m d}^{-}$ \\
\hline 0 & 1100 & 650 & 925 & 0.19 & 0.30 \\
20 & 2400 & 1150 & 1925 & 0.25 & 0.40 \\
40 & 3400 & 1700 & 2750 & 0.23 & 0.38 \\
60 & 4200 & 2200 & 3425 & 0.23 & 0.36 \\
80 & 5000 & 2800 & 4125 & 0.21 & 0.32 \\
100 & 5800 & 3200 & 4675 & 0.24 & 0.32 \\
\hline \hline
\end{tabular}




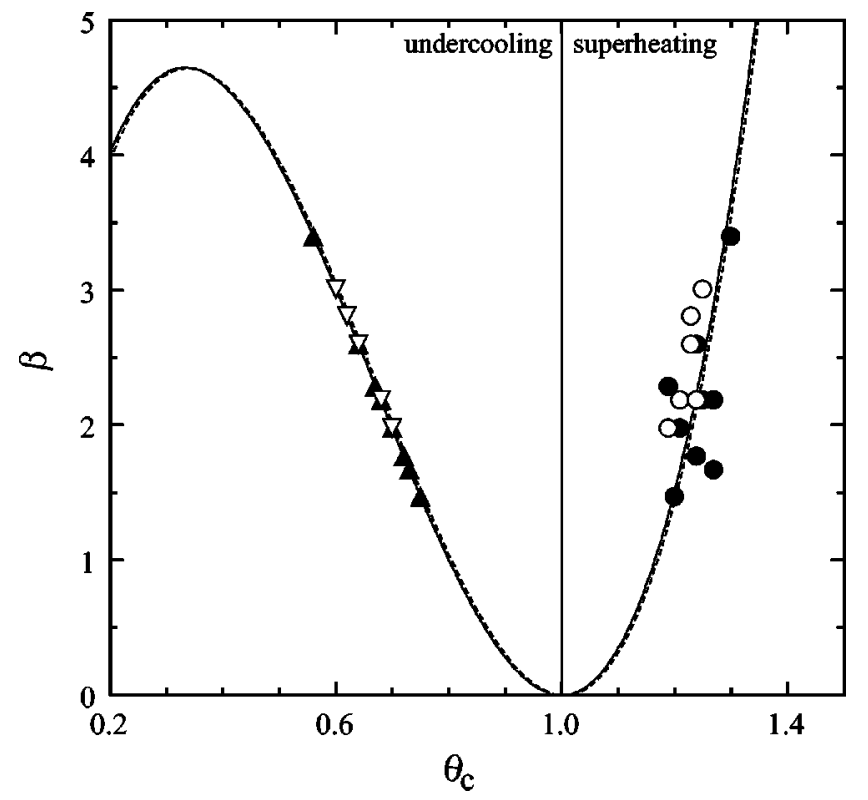

FIG. 5. Deduced energy barrier $\beta$ vs maximum undercooling and superheating acheived $\left(\theta_{c}\right)$ in MD simulations for close-packed metals. Open symbols are for $\mathrm{Al}$ at $P=0,20,40,60,80$, and 100 GPa (Table VI) and solid symbols for other elements (ambient pressure, Table V) simulated with the qSC force field. The solid curve is the plot of $\beta=\left(A_{0}-b \log _{10} Q\right) \theta_{c}\left(\theta_{c}-1\right)^{2}$ with $Q=10^{12} \mathrm{~K} / \mathrm{s}$. The dashed curve is the best fit of simulations to the same functional form [i.e., $\beta=A(Q) \theta_{c}\left(\theta_{c}-1\right)^{2}$ ].

systems investigated as self-consistent and examine the consistency of the MD simulation with the maximum superheating-undercooling systematics. To serve this purpose, it would be ideal if $\gamma_{s l}$ were calculated from MD simulations. In that case, $\beta$ for a specific system under certain $P$ and $T$ conditions could be obtained directly as $\Delta H_{m}$ and $T_{m}$ can be calculated readily from MD, and the maximum superheating and undercooling achieved in MD can be compared directly with the systematics. As $\gamma_{s l} \sim 0.01-0.1 \mathrm{~J} / \mathrm{m}^{2}$ in order of magnitude, the contribution from solid-liquid interfaces to the total energy of the system should be negligible. The twophase technique described above is not appropriate for simulating $\gamma_{s l}$. An alternative way to check the consistency is to employ both $\Theta_{m d}^{+}$and $\Theta_{m d}^{-}$. Suppose that the maximum undercooling (or superheating) can be described by the systematics [Eq. (7)]. Given $\Theta_{m d}^{-}$(or $\Theta_{m d}^{+}$), the material property $\beta$ of the system with the prescribed force field can be then obtained from Eq. (7). If $\beta$ is obtained from $\Theta_{m d}^{-}$(Tables $\mathrm{V}$ and VI), the predicted superheating $\left(\Theta_{n s}^{+}\right)$at a comparable heating rate $Q \sim 10^{12} \mathrm{~K} / \mathrm{s}$ can be checked against results from simulations. Both $\Theta_{m d}^{-}$and $\Theta_{m d}^{+}$along with $\beta$ (from $\Theta_{m d}^{-}$) were fitted to the same functional form as the systematics [Eq. (6)], and we found excellent agreement between the fitting to the MD results (dashed curve) and the systematics (solid curve, Fig. 5). Note that the MD results at both ambient pressure and high pressures (for $\mathrm{Al}$ ) are included in Fig. 5. Thus, the maximum superheating and undercooling achieved in MD simulations with prescribed force fields are consistent with the maximum superheating-undercooling systematics developed from undercooling experiments. The abnormal behavior of undercooled $\mathrm{Pb}$ is the only exception which does not comply with the systematics, possibly due to an ill-posed force field, or the atomic size of $\mathrm{Pb}$.

We have demonstrated excellent agreement between MD simulations and the maximum superheating-undercooling systematics. Thus the empirical systematics are validated at the atomic level. A direct application is to predict the interfacial energy $\gamma_{s l}$. The heat of fusion $\left(\Delta H_{m}\right)$ can be obtained from the enthalpy $(H)$ difference between solid and liquid from single-phase simulations, $T_{m}$ (i.e., $T_{2, m}$ ) from twophase simulations, and $\beta$ from either $\Theta_{m d}^{+}$or $\Theta_{m d}^{-}$and the systematics; thus $\gamma_{s l}$ can be derived (Table V).

\section{SUPERHEATING IN ULTRAFAST DYNAMIC EXPERIMENTS}

In shock-wave loading such as planar impact experiments, the rise time of the shock in nonporous solids is of nanosecond order. For shocks strong enough to induce melting in typical solids, the temperature increase is of order $10^{3} \mathrm{~K}$, so $Q \sim 10^{12} \mathrm{~K} / \mathrm{s}$. The solid is heated internally as the shock front advances, and surface melting may be suppressed in compression. The long-range order of shocked solid can persist because the temperature rises faster than the atoms can rearrange to melt. The kinetics inherent in the solid-liquid transition may play an important role at the time scale of the shock front, thus allowing significant superheating to occur.

Techniques employed to detect shock melting include sound speed and temperature measurements of the shocked state. ${ }^{4}$ When the solid melts, the sound speed drops from the longitudinal to the bulk value due to the loss of rigidity. Similarly, the latent heat of fusion decreases the temperature reached behind a shock inducing melting. Other techniques such as transient electron diffraction are important diagnostics to detect melting from the loss of long-range order of laser-irradiated crystal. $^{6}$ But real-time structure measurements are scarce due to technical challenges. Melting might not be recognizable in a pressure-density Hugoniot function, because the density change due to melting at high pressures may be small. Changes in the slope of the shock-velocityparticle-velocity relationship may serve as a complement to other techniques.

A typical example of shock-induced superheating is shown in Fig. 6 for $\mathrm{CsBr}^{4}$ For shocks above $\sim 38 \mathrm{GPa}$, there is a decrease in sound speed and shock temperature, signaling melting. If the $T-P$ Hugoniot states achieved thermodynamic equilibrium and were represented by abcde where $b c d$ coincides with the equilibrium melting curve, the shocked solid would melt at $b$ and the successive Hugoniot states lie along the equilibrium phase boundary $b c d$. As the measured $T-P$ Hugoniot function states lie along $a b c^{\prime} d e$, it appears that the shocked solid is superheated metastably to a maximum $T_{c^{\prime}}$ before it melts. In this case, the liquid Hugoniot terminates on the equilibrium melting curve. The degree of superheating near the transition pressure $P_{c}$ on the Hugoniot, $\Theta_{H}^{+}=T_{c^{\prime}} / T_{c}-1$. For CsBr, $\Theta_{H}^{+} \sim 0.19$. A significant number of shock melting experiments with sound speed and temperature diagnostics have been conducted on alkali halides ${ }^{4}$ $\left(\mathrm{CsBr}\right.$ and $\mathrm{KBr}$ ), transition metals ${ }^{5,7}$ ( $\mathrm{Fe}$ and $\mathrm{V}$ ) and 


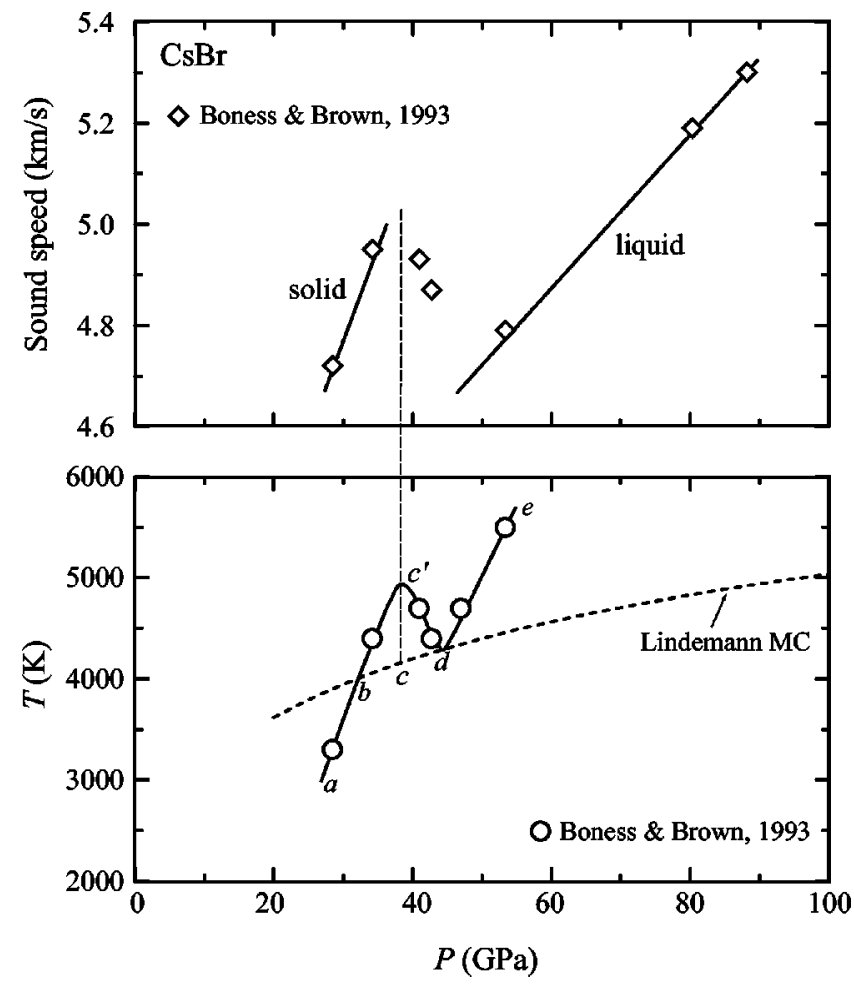

FIG. 6. Typical experimental example of melting under shock wave loading: $\mathrm{CsBr}$ (Ref. 4). Simultaneous measurements of shockstate sound speed (upper panel) and temperature (lower panel) demonstrate simultaneous drop in sound speed and shock temperature, signaling melting of shocked crystal at higher shock pressures than $P_{c}$ (the long dashed curve). Solid curves indicate the Hugoniot states. The dashed curve is the Lindemann melting curve (MC) (Ref. 31). $b c^{\prime}$ segment denotes superheated states.

silicates ${ }^{3,40}$ (fused silica, quartz and $\mathrm{Mg}_{2} \mathrm{SiO}_{4}$ ); similar superheating-melting behavior deviating from equilibrium melting has been observed. The results from planar shock wave loading are summarized in Table VII.

Heating rates of $\sim 10^{12} \mathrm{~K} / \mathrm{s}$ may be obtained using intense laser irradiation, depending on energy deposited, irradiation time, and material properties. In laser irradiation experiments, real-time crystal structure information can be obtained from transient electron diffraction and the temperature from calibration, mass spectometry, or inferred. Significant superheating has been observed ${ }^{6,41,43,42}$ in laser-irradiated Al, $\mathrm{Pb}(111), \mathrm{Bi}(0001)$, and GaAs (Table VII).

Superheating-melting behavior appears to be the dominant feature in shock melting experiments (including laser irradiation). The observed superheating compares favorably to the prediction of the superheating systematics at $Q$ $\sim 10^{12} \mathrm{~K} / \mathrm{s}$ (Table VII). We assumed that $\beta$ remains the same at high pressures (where solid-solid phase changes or chemical decomposition may occur) for planar impact experiments. Shock loading on $\mathrm{Mg}_{2} \mathrm{SiO}_{4}$ demonstrates unusual superheating, ${ }^{40}$ larger than the prediction for $\mathrm{Ga}(0.43)$ at a similar heating rate. The result could have been complicated by unknown effects of phase changes and decomposition at high pressures. Pressure was assumed to be ambient in laser irradiation experiments by these authors. Uncertainties in de-
TABLE VII. Superheating achieved in ultrafast dynamic experiments: planar shock-wave loading and intense laser irradiation. Note that materials may be subjected to phase changes and decomposition at high pressures. See text for references.

\begin{tabular}{lccccc}
\hline \hline $\begin{array}{l}\text { Starting } \\
\text { Material }\end{array}$ & $\begin{array}{c}P_{c} \\
(\mathrm{GPa})\end{array}$ & $\begin{array}{c}T_{c} \\
(\mathrm{~K})\end{array}$ & $\begin{array}{c}T_{c^{\prime}}{ }^{\mathrm{a}} \\
(\mathrm{K})\end{array}$ & $\Theta_{H}^{+}$ & $\Theta_{n s}^{+}$ \\
\hline \multicolumn{5}{c}{ Planar Impact } \\
$\mathrm{Fe}$ & 270 & 5800 & 7250 & 0.25 & 0.28 \\
$\mathrm{~V}$ & 220 & 6150 & 7600 & 0.24 & 0.23 \\
$\mathrm{CsBr}$ & 38 & 4150 & 4950 & 0.19 & 0.20 \\
$\mathrm{KBr}$ & 28 & 3500 & 4200 & 0.20 & 0.18 \\
Fused quartz & 70 & 4500 & 5300 & 0.18 & - \\
Quartz & 113 & 4800 & 6100 & 0.27 & $0.34^{\mathrm{b}}$ \\
$\mathrm{Mg}_{2} \mathrm{SiO}_{4}$ & 130 & 4300 & 7000 & 0.63 & - \\
$\mathrm{Al}$ & 0 & Laser irradiation & & \\
$\mathrm{Bi}(0001)$ & 0 & 533 & $1300^{\mathrm{c}}$ & 0.39 & 0.20 \\
$\mathrm{~Pb}(111)$ & 0 & 601 & 721 & 0.20 & 0.37 \\
$\mathrm{GaAs}$ & 0 & 1511 & 2061 & 0.36 & - \\
\hline \hline
\end{tabular}

${ }^{\mathrm{a}}$ Estimated from the $T-P$ Hugoniot.

${ }^{\mathrm{b}}$ Value for quartz at ambient pressure (Ref. 2).

${ }^{\mathrm{c}}$ Value for irradiation flux of $7 \mathrm{~mJ} / \mathrm{cm}^{2}$ is adopted (Ref. 7).

termining the temperature could also contribute to the estimation of superheating. Despite these uncertainties, it is clear that solids can be superheated substantially beyond the equilibrium melting point by ultrafast heating, and the amount of superheating is comparable to predictions of the superheating systematics. The significance of heating rates lies in the fact that ultrafast heating is crucial to achieving superheating.

By considering shock-induced superheating (Table VII), equilibrium melting curves at high pressures were constructed based on the Lindemann law for silicates, alkali halides, and transition metals. ${ }^{31}$ The static DAC and shockwave results are in accord for silica and alkali halides. ${ }^{31}$ But significant discrepancies exist for transition metals. A sys-

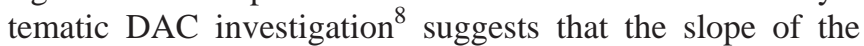
melting curve $d T_{m} / d P$ for transition metals becomes nearly zero at $\sim 100 \mathrm{GPa}$. If we extrapolate the DAC melting curves $^{8}$ for Fe, V, Mo, W, and Ta to 200-400 GPa where shock melting occurs, shock temperature measurements and calculations $^{5,7,44-46}$ would indicate $\Theta_{H}^{+} \sim 0.7-2.0$. These large values of superheating are not consistent with the superheating systematics developed above, even when the uncertainties are taken into account. The discrepancies could be reconciled by possible solid-solid phase transitions at high pressures. $^{47}$

\section{CONCLUSION}

The maximum superheating and undercooling $\left(\theta_{c}\right)$ depend on the material parameter $\beta$ internally and on the heating (cooling) process (i.e., $Q$ ) externally. The $\theta_{c}-\beta-Q$ systematics for the maximum superheating and undercooling were established as $\beta=\left(A_{0}-b \log _{10} Q\right) \theta_{c}\left(1-\theta_{c}\right)^{2}$, based 
on classical nucleation theory and undercooling experiments. $\beta$ is weakly dependent on pressure and varies between 0.2 and 8.2 for the elements and compounds investigated. This range of $\beta$ can be regarded as typical of solids in general. Solids can be superheated by $(0.05-0.35) T_{m}$ and $(0.08-0.43) T_{m}$ (i.e., less than $\left.0.5 T_{m}\right)$ at 1 and $10^{12} \mathrm{~K} / \mathrm{s}$, respectively. The largest possible undercooling $\left(\Theta^{-}=2 / 3\right)$ was not observed in the data investigated, but should be achievable by increasing the cooling rate. Materials with $\beta>\beta_{\max }$ $=\frac{4}{27}\left(A_{0}-b \log _{10} Q\right)$ should not crystallize at any cooling rate $Q$.

Systematic molecular dynamics simulations were conducted on close-packed metals using single- and two-phase simulation techniques. The maximum superheating and undercooling predicted was consistent with the systematics established empirically, thus validating the systematics at the level of interatomic interactions. The heating rate is crucial to achieving appreciable superheating experimentally. We demonstrated that superheating achieved in ultrafast dynamic experiments, such as planar shock-wave loading and intense laser irradiation, agrees with the predictions of the $\theta_{c}-\beta-Q$ systematics.

Catastrophic nucleation near the maximum superheating and undercooling $\left(\theta_{c}\right)$ dominates the nucleation process.
Such a catastrophe is driven by free energy and can be regarded as a kinetic limit. Previous efforts in MD simulations attempted to relate the thermodynamic melting to the Lindemann criterion (vibrational instability) and Born (mechanical) instability. ${ }^{48,49}$ In MD simulations of a Lennard-Jones fcc system, superheating of $\Theta_{m d}^{+} \sim 0.20$ corresponds to Lindemann's parameter $\delta_{L} \sim 0.22$ (fractional root-mean-square displacement) and near-zero shear moduli of a bulk system. ${ }^{49}$ It is not surprising that both criteria are satisfied at the kinetic limit of superheating. We have validated the systematics of the kinetic limit for melting and freezing at the atomic level and demonstrated that superheating achieved in dynamic experiments agrees with the systematics. But a universal relationship between the kinetic limit, Lindemann's criterion, and the Born instability needs to be established and quantified from first principles.

\section{ACKNOWLEDGMENTS}

This work has been supported by U.S. NSF Grant No. EAR-0207934 (T.J.A.). S.N.L. is sponsored by Los Alamos National Laboratory, contribution No. 8932, GPS Division, California Institute of Technology.
*Current address: P-24 Plasma Physics, Los Alamos National Laboratory, Los Alamos, NM 87545. Electronic address: sluo@lanl.gov

${ }^{1}$ K.F. Kelton, Solid State Phys. 45, 75 (1991).

${ }^{2}$ D.R. Uhlmann, J. Non-Cryst. Solids 41, 347 (1980).

${ }^{3}$ G.A. Lyzenga, T.J. Ahrens, and A.C. Mitchell, J. Geophys. Res. 88, 2431 (1983).

${ }^{4}$ D.A. Boness and J.M. Brown, Phys. Rev. Lett. 71, 2931 (1993).

${ }^{5}$ C.Y. Yoo, N.C. Holmes, M. Ross, D.J. Webb, and C. Pike, Phys. Rev. Lett. 70, 3931 (1993).

${ }^{6}$ S. Williamson, G. Mourou, and J.C.M. Li, Phys. Rev. Lett. 52, 2364 (1984).

${ }^{7}$ C. Dai, X. Jin, X. Zhou, J. Liu, and J. Hu, J. Phys. D 34, 3064 (2001).

${ }^{8}$ D. Errandonea, B. Schwager, R. Ditz, C. Gessmann, R. Boehler, and M. Ross, Phys. Rev. B 63, 132104 (2001).

${ }^{9}$ M.P. Allen and D.J. Tildesley, Computer Simulation of Liquids (Clarendon, Oxford, 1987).

${ }^{10}$ J.R. Morris, C.Z. Wang, K.M. Ho, and C.T. Chan, Phys. Rev. B 49, 3109 (1994).

${ }^{11}$ Z.H. Jin and K. Lu, Philos. Mag. Lett. 78, 29 (1998).

${ }^{12}$ A. Strachan, T. Çağın , and W.A. Goddard III, Phys. Rev. B 60, 15084 (1999).

${ }^{13}$ S.-N. Luo, T. Çağın , A. Strachan, W.A. Goddard III, and T.J. Ahrens, Earth Planet. Sci. Lett. 202, 147 (2002).

${ }^{14}$ F.A. Lindemann, Phys. Z. 11, 609 (1910).

${ }^{15}$ M. Born, J. Chem. Phys. 7, 591 (1939).

${ }^{16}$ J.H. Wang, J. Li, S. Yip, D. Wolf, and S.R. Phillpot, Physica A 240, 396 (1997).

${ }^{17}$ H.J. Fecht and W.L. Johnson, Nature (London) 334, 50 (1983).

${ }^{18}$ J.L. Tallon, Nature (London) 342, 658 (1989).

${ }^{19}$ K. Lu and Y. Li, Phys. Rev. Lett. 80, 4474 (1998).
${ }^{20}$ B. Rethfeld, K. Sokolowski-Tinten, D. von der Linde, and S.I. Anisimow, Phys. Rev. B 65, 092103 (2002).

${ }^{21}$ S.-N. Luo and T.J. Ahrens, Appl. Phys. Lett. 82, 1836 (2003).

${ }^{22}$ H.B. Singh and A. Holz, Solid State Commun. 45, 985 (1983).

${ }^{23}$ D. Turnbull, J. Chem. Phys. 20, 411 (1952).

${ }^{24}$ J.W. Christian, The Theory of Transformation in Metals and Alloys (Pergaman, New York, 1965).

${ }^{25}$ A.G. Walton, in Nucleation, edited by A.C. Zettlemoyer (Marcel Dekker, New York, 1969), p. 225.

${ }^{26}$ V.I. Motorin and S.L. Musher, J. Chem. Phys. 81, 465 (1984).

${ }^{27}$ Y. Shao and F. Spaepen, J. Appl. Phys. 79, 2981 (1996).

${ }^{28}$ D.A. Porter and K.E. Easterling, Phase Transformations in Metals and Alloys (Van Nostrand Reinhold International, Co. Ltd., Wokingham, Berkshire, England, 1981).

${ }^{29}$ R.L. Davidchack and B.B. Laird, Phys. Rev. Lett. 85, 4751 (2000).

${ }^{30}$ W.G. Hoover and F.H. Ree, J. Chem. Phys. 49, 3609 (1968).

${ }^{31}$ S.-N. Luo and T. J. Ahrens, Phys. Earth Planet. Inter. (to be published).

${ }^{32}$ M.C. Weinberg, J. Non-Cryst. Solids 83, 98 (1986).

${ }^{33}$ D. Frenkel and B. Smit, Understanding Molecular Dynamics (Academic, San Diego, 1996).

${ }^{34}$ A. Strachan, T. Çağın, O. Gulseren, S. Mukherjee, R. E. Cohen, and W. A. Goddard III (unpublished).

${ }^{35}$ A.B. Belonoshko, R. Ahuja, and B. Johansson, Phys. Rev. Lett. 84, 3638 (2000).

${ }^{36}$ A.P. Sutton and J. Chen, Philos. Mag. Lett. 61, 139 (1990).

${ }^{37}$ Y. Kimura, Y. Qi, T. Çağın, and W. A. Goddard III (unpublished).

${ }^{38}$ W.G. Hoover, Phys. Rev. A 31, 1695 (1985).

${ }^{39}$ M. Parinello and A. Rahman, J. Appl. Phys. 52, 7182 (1981).

${ }^{40}$ K.G. Holland and T.J. Ahrens, Science 275, 1623 (1997).

${ }^{41}$ J.W. Herman and H.E. Elsayed-Ali, Phys. Rev. Lett. 69, 1228 (1992). 
${ }^{42}$ N. Fabricius, P. Hermers, and D. von der Linde, Solid State Commun. 58, 239 (1986).

${ }^{43}$ E.A. Murphy, H.E. Elsayed-Ali, and J.W. Herman, Phys. Rev. B 48, 4921 (1993).

${ }^{44}$ J.W. Shaner, J.M. Brown, and R.G. McQueen, in High Pressure in Science and Technology, edited by C. Homan, R.K. MacCrone, and E. Whalley, Mater. Res. Soc. Symp. Proc. No. 22 (Materials Research Society, Pittsburgh, 1984), p. 137.
${ }^{45}$ R.S. Hixson, D.A. Boness, J.W. Shaner, and J.A. Moriarty, Phys. Rev. Lett. 62, 637 (1989).

${ }^{46}$ R.S. Hixson and J.N. Fritz, J. Appl. Phys. 71, 1721 (1992).

${ }^{47}$ J.M. Brown, Geophys. Res. Lett. 28, 4339 (2001).

${ }^{48}$ S.R. Phillpot, S. Yip, and D. Wolf, Comput. Phys. 3, 20 (1989).

${ }^{49}$ Z.H. Jin, P. Gumbsch, K. Lu, and E. Ma, Phys. Rev. Lett. 87, 055703 (2001). 